\title{
Silencing Kif2a induces apoptosis in squamous cell carcinoma of the oral tongue through inhibition of the PI3K/Akt signaling pathway
}

\author{
KETAO WANG $^{1 *}$ CHANGLONG LIN $^{1 *}$, CHENGQIN WANG $^{2}$, QIANQIAN SHAO ${ }^{3}$, WENJUAN GAO ${ }^{3}$, \\ BINGFENG SONG ${ }^{3}$, LEI WANG ${ }^{1}, \mathrm{XIAOBIN} \mathrm{SONG}^{1}, \mathrm{XUN} \mathrm{QU}^{3}$ and FENGCAI WEI ${ }^{1}$
}

${ }^{1}$ Department of Oral and Maxillofacial Surgery and Institution of Dental Medicine, Qilu Hospital, Shandong University, Jinan,
Shandong 250012; ${ }^{2}$ Department of Pathology, Medical College of Qingdao University, Qingdao, Shandong 266000;
${ }^{3}$ Institute of Basic Medical Sciences, Qilu Hospital, Shandong University, Jinan, Shandong 250012, P.R. China

Received March 2, 2013; Accepted October 28, 2013

DOI: $10.3892 / \mathrm{mmr} .2013 .1804$

\begin{abstract}
Previous studies have demonstrated that the overexpression of Kif $2 \mathrm{a}$ is involved in the progression, invasion and metastasis of squamous cell carcinoma of the oral tongue (SCCOT). Few studies have reported the correlation between Kif2a and apoptosis of tumor cells and which signaling pathways Kif2a is involved in remains unclear. The phosphatidylinositol-3-kinase (PI3K)/protein kinase B (Akt) pathway is frequently activated in many types of human cancer. The aim of the present study was to investigate the effects of downregulation of Kif2 expression on the P13K/Akt pathway in Tca8113 cells to determine whether silencing of Kif2 inhibits the P13K/Akt pathway, resulting in cell apoptosis. siRNA vector was constructed, western blot analysis was used to determine RNA interference and flow cytometry was used to determine promotion of Tca8113 cell apoptosis. The results revealed that silencing Kif $2 \mathrm{a}$ induces apoptosis and decreases the mRNA and protein level of PI3K, Akt and B-cell lymphoma 2 (Bcl-2) in Tca8113 cells. The PI3K-specific agonist insulin-like growth factor 1 (IGF-1) eliminated the upregulation of apoptosis of Tca8113-Kif2a cells by phosphorylation of Akt. The results suggest that silencing Kif2a induces tumor cell apoptosis, at least partially, through the PI3K/Akt signaling pathway.
\end{abstract}

Correspondence to: Professor Xun Qu, Institute of Basic Medical Sciences, Qilu Hospital, Shandong University, 107 WenhuaXi Road, Jinan, Shandong 250012, P.R. China

E-mail: quxun@sdu.edu.cn

Professor Fengcai Wei, Institution of Dental Medicine, Shandong University, 107 Wenhua Xi Road, Jinan, Shandong 250012, P.R. China E-mail: weifengcai@yahoo.cn

*Contributed equally

Key words: kinesin-13 family 2a, phosphatidylinositol-3-kinase, protein kinase B, squamous cell carcinoma of the oral tongue, apoptosis

\section{Introduction}

The human genome has three distinct genes encoding members of the kinesin-13 family, known as Kif2a (chromosome 5q12), Kif2b (chromosome 17q22) and MCAK/Kif2c (chromosome 1p34). Kif2a is essential for bipolar spindle assembly and chromosome movement (1). It localizes to spindle poles in human cells and cells lacking Kif2a form monopolar spindles instead of bipolar spindles in mitosis (2), leading to arrest of cell cycle progression. A previous study demonstrated that the overexpression of Kif $2 \mathrm{a}$ is involved in the progression, invasion and metastasis of squamous cell carcinoma of the oral tongue (SCCOT) (3). However, as yet, very little information is available on the correlation between Kif $2 \mathrm{a}$ and apoptosis of tumor cells. Additionally, the signaling pathways that Kif2a is involved in remain to be determined.

Phosphatidylinositol-3-kinase (PI3K) is a lipid kinase that generates second messengers involved in the regulation of many cell functions including proliferation, survival and invasion (4). One of its major effectors is protein kinase B (Akt). Results of previous studies have shown that the PI3K/Akt pathway is frequently activated in many types of human cancer, including head and neck squamous cell carcinoma (5-8), and that phospho-Akt and Akt2 were overexpressed in oral squamous cell carcinoma (9). Therefore, the evidence suggests that the PI3K/Akt signaling pathway is likely associated with the cell proliferation, apoptosis and migration of SCCOT. However, the mechanism responsible for PI3K/Akt activation in SCCOT has yet to be determined.

SCCOT is a common tumor of the head and neck (10). Since Kif2a overexpression and PI3K/Akt activation are frequent events in oral squamous cell carcinoma and are associated with tumor progression of SCCOT, we investigated whether there is any correlation between Kif $2 \mathrm{a}$ and PI3K/Akt signaling. We examined the effects of downregulated Kif2a expression on the PI3K/Akt pathway and the pro-apoptotic role of silencing Kif $2 \mathrm{a}$ in SCCOT cells. In addition, we studied the effects of a pharmaceutical inhibitor and agonist of PI3K on the anti-apoptotic function of Kif2a in SCCOT cells. The 
results suggest that silencing Kif2a inhibits the PI3K/Akt signaling pathway, leading to cell apoptosis.

\section{Materials and methods}

Cell culture. Human tongue squamous cell carcinoma cell lines (Tca8113 cells) were purchased from the Culture Collection of Chinese Academy of Science (Shanghai, China) and routinely cultured in Dulbecco's modified Eagle's medium (Gibco-BRL, Carlsbad, CA, USA) containing 10\% fetal bovine serum (Gibco-BRL), penicillin $(100 \mathrm{U} / \mathrm{ml})$ and streptomycin $(100 \mu \mathrm{g} / \mathrm{ml})$ at $37^{\circ} \mathrm{C}$ in a humidified air atmosphere containing $5 \% \mathrm{CO}_{2}$.

Silencing Kif2a gene using small interfering RNA (si-RNA). si-RNA expression vector was constructed by introducing synthetic double-stranded oligonucleotides (Kif2a: 5'-CACCGGCAAAGAGATTGACCTGGTTCAAGAGACC AGGTCAATCTCTTTGCCTTTT TTG-3'; nonsense control: 5'-CACCGTTCTCCGAACGTGTCACGTCAAGAGATTAC GTGACACGTTCGGAGAATTTTTTG-3') synthesized by GeneChem (Shanghai, China) and inserted into the pGPU/ GFP/Neo vector (Shanghai GenePharma, Ltd., Shanghai, China). Authenticity of the constructs was confirmed by sequencing Tca8113 cells that were planted in six-well plates in Opti-MEM (Invitrogen Life Technologies, Carlsbad, CA, USA) until the cells reached $40 \%$ confluence. The cells were transfected with the kif2a si-RNA (si-Kif2a) or nonsense si-RNA (si-NC) (100 nmol/l) premixed with the Lipofectamine $^{\text {TM }} 2000$ (Invitrogen Life Technologies) in Opti-MEM. Mock transfection with Lipofectamine ${ }^{\mathrm{TM}} 2000$ only was also included as a control. Six hours after transfection, the cells were placed in fresh complete medium without penicillin and streptomycin. Human insulin-like growth factor 1 (IGF-1) was purchased from InvivoGen (San Diego, CA, USA) and Peprotech, Inc. (Rocky Hill, NJ, USA).

Western blot analysis. Western blot analysis was performed as previously described (3). In brief, cells were collected and lysed in 1\% NP-40 lysis buffer. Then cell extract was resolved on $10 \%$ polyacrylamide gels using minigel apparatus and transferred to a polyvinylidene fluoride membrane. The membrane was blotted with rabbit anti-Kif2a polyclonal antibodies at dilution of 1:10,000 (Abcam, Cambridge, UK) for $2 \mathrm{~h}$ at room temperature. Blots were then exposed to horseradish peroxidase-conjugated goat anti-rabbit $\lg \mathrm{G}$ (dilution 1:10,000), followed by development using an electrochemiluminescence reagent.

Real-time reverse transcription-polymerase chain reaction (RT-PCR) analysis. After Tca8113 cells were collected, total RNA was extracted using the TRIzol (Invitrogen Life Technologies) method as recommended by the manufacturer. RNA was reverse transcribed by the First Strand cDNA Synthesis kit (Fermentas, Burlington, Canada). According to the protocol recommended by Takara SYBR Premix Ex Taq $^{\mathrm{TM}}$ (Takara Bio, Inc., Shiga, Japan), real-time quantitative PCR analysis was performed using a LightCycler 2.0 (Roche Diagnostics, Basel, Switzerland). Thermal cycling parameters were as follows: an initial incubation of $95^{\circ} \mathrm{C}$ for $30 \mathrm{sec}$ and
Table I. Primer sequences of genes.

\begin{tabular}{llc}
\hline Gene & \multicolumn{1}{c}{\begin{tabular}{c}
\multicolumn{1}{c}{ Primer sequence } \\
$\left(5^{\prime}-3^{\prime}\right)$
\end{tabular}} & $\begin{array}{c}\text { Product size } \\
(\mathrm{bp})\end{array}$ \\
\hline 3-actin & $\begin{array}{l}\text { F: CGTTGACATCCGTAAAGACC } \\
\text { R: TAGAGCCACCAA TCCACAC }\end{array}$ & 176 \\
PI3K & F: CATCACTTCCTCCTGCTCTAT & 377 \\
& R: CAGTTGTTGGCAATCTTCTTC & \\
Akt & F: TGCATTGCCGAGTCCAGAA & 139 \\
& R: GCATCCGAGAAA CAAAACATCA & \\
Bcl-2 & F: GCAGAGATGTCCAGTCAG & 129 \\
& R: CCCACCGAACTCAAAGAAGG & \\
Bax & F: ATGGGCTGGACACTGGACTTC & 146 \\
& R: GAGCGAGGCGGTGAGGAC & \\
\hline
\end{tabular}

F, forward; R, reverse; PI3K, phosphatidylinositol-3-kinase; Akt, protein kinase B; Bcl-2, B-cell lymphoma 2; Bax, Bcl-2-associated X protein.

then 40 cycles of $95^{\circ} \mathrm{C}$ for $5 \mathrm{sec}, 55^{\circ} \mathrm{C}$ for $20 \mathrm{sec}$ and $72^{\circ} \mathrm{C}$ for $15 \mathrm{sec}$. Forward and reverse primer sequences for PI3K, Akt, B-cell lymphoma 2 (Bcl-2), Bcl-2-associated X protein (Bax) and $\beta$-actin are shown in Table I.

Annexin V-fluorescein isothiocyanate (FITC) assay. To assess the degree of apoptosis of different groups of Tca8113 cells, the extent of Annexin V-FITC/ propidium iodide (PI) staining was determined by flow cytometry using the Annexin V/PI staining kit from Bender MedSystems (Vienna, Austria). Samples were read on an Epics XL-MCL flow cytometer (Beckman Coulter, Brea, FL, USA) and were analyzed by WinMDI 2.8 software.

GeneChip hybridization and data analysis. GeneChip hybridization and data analysis were performed by KangChen Bio-tech (Shanghai, China). Purified and fragmented cRNA probes were hybridized onto Affymetrix Human Genome-U133A 2.0 GeneChips (Affymetrix, Inc., Santa Clara, CA, USA). Each RNA pool was hybridized to an individual chip and hybridization was performed in the presence of herring sperm DNA $(0.1 \mathrm{mg} / \mathrm{ml}$; Sigma-Aldrich, St. Louis, MO, USA) for $16 \mathrm{~h}$ at $45^{\circ} \mathrm{C}$. Chips were then washed, stained with streptavidinphycoerythrin and scanned with a confocal microscope scanner (GeneArray Scanner 2500; Hewlett-Packard, Palo Alto, CA, USA) according to Affymetrix guidelines. The standard Affymetrix analysis software algorithms (Microarray Suite 5.0) were used for data capturing, which selects the spots representative of a transcript and subtracts the background from the significant signals. The images from the scanned chip were processed on GeneSpring 7.2 software (Silicon Genetics, Redwood City, CA, USA). Gene expression data for each replicate experiment were normalized using the 'per chip normalization' and 'per gene normalization' algorithms implemented in the GeneSpring program. Genes with expression levels altered by $\geq 1$.5-fold in Kif2a silenced relative to si-NC samples were considered to be differentially expressed. The significance of gene expression differences between the two experimental conditions was calculated using 
A
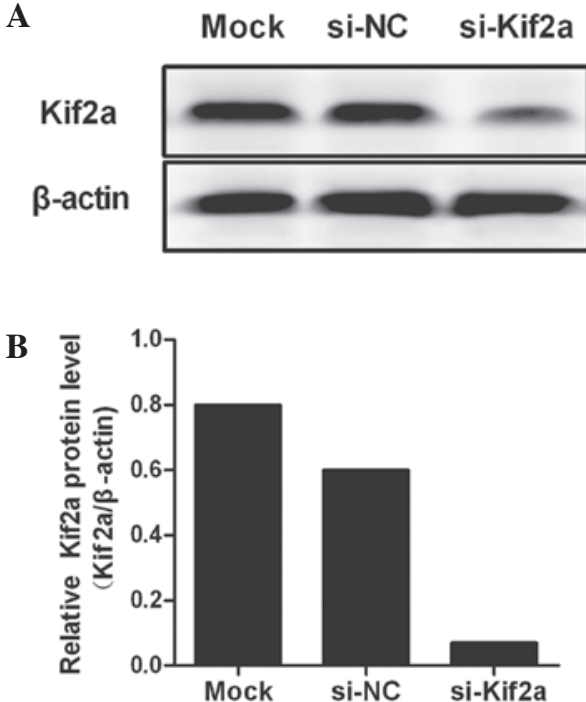

C Mock si-NC si-Kif2a

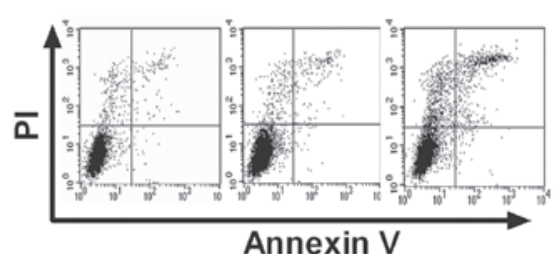

D

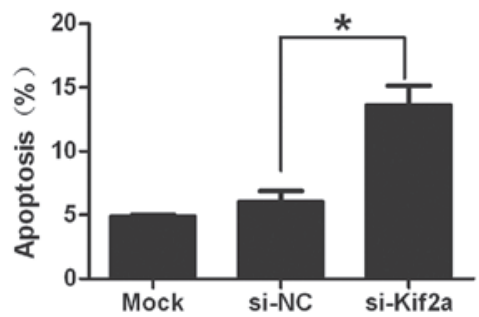

Figure 1. Effects of kif2a si-RNA treatment on Tca8113 cells. (A) Tca8113 cells were transfected with the lipofectamine only (Mock), kif2a si-RNA (si-Kif2a) or nonsense si-RNA (si-NC). After $72 \mathrm{~h}$, the expression of Kif2a protein in the group transfected with kif2a si-RNA was decreased compared with the other two groups ( $\mathrm{P}=0.022)$. (B) Tubulin was used as a control. Mock was used as a negative control. Data are expressed as the gray-scale ratio of Kif2a protein relative to that of tubulin. (C and D) Kif2a si-RNA promoted the apoptosis of Tca8113 cells. The ratio of apoptosis of Tca8113-Kif2a cells was increased more clearly than in the other two groups ( $\mathrm{P}<0.05)$. The data are presented as the means \pm SD. Experiments were repeated $\geq 3$ times.

\begin{tabular}{|l|l|l|}
\hline UniGene ID & Symbol & Gene title \\
\hline Hs.631546 & BAX & Homo sapiens BCL2-associated X protein \\
\hline Hs.529957 & SEC63 & Homo sapiens SEC63 homolog (S. cerevisiae) \\
\hline Hs.521640 & RAD23B & Homo sapiens RAD23 homolog B (S. cerevisiae) \\
\hline Hs.131431 & EIF2AK2 & Homo sapiens eukaryotic translation initiation factor 2-alpha kinase 2 \\
\hline Hs.132753 & FBXO2 & Homo sapiens F-box protein 2 \\
\hline Hs.518602 & WFS1 & Homo sapiens Wolfram syndrome 1 (wolframin) \\
\hline Hs.522817 & BCAP31 & Homo sapiens B-cell receptor-associated protein 31 \\
\hline Hs.75890 & MBTPS1 & Homo sapiens membrane-bound transcription factor peptidase, site 1 \\
\hline Hs.567533 & YOD1 & Homo sapiens YOD1 OTU deubiquinating enzyme 1 homolog (S. cerevisiae) \\
\hline Hs.465295 & LMAN1 & Homo sapiens lectin, mannose-binding, 1 \\
\hline
\end{tabular}

Fold change

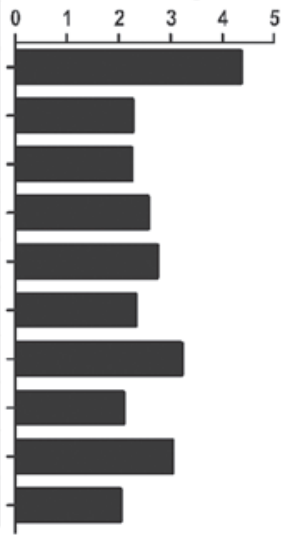

Figure 2. Gene expression changes in Tca8113-Kif2a and Tca8113-nonsense cells. Of these genes, the expression of the BAX gene was upregulated $>4$-fold in Tca8113-Kif2a cells.

a one-way analysis of variance. Biological theme analyses were conducted by Expression Analysis Systematic Explorer and the Database for Annotation, Visualization and Integrated Discovery (DAVID) 2.0 program.

Statistical analysis. Statistical analysis was performed using the SPSS 16.0 software package for Windows. Values are presented as the means \pm standard deviation (SD). The Student's t-test was used for paired data that were normally distributed. $\mathrm{P}<0.05$ was considered to indicate a statistically significant difference.

\section{Results}

Kif 2 a expression is suppressed by si-Kif $2 a$. RNA interference targeting Kif2a was determined by western blot analysis. Reduction of Kif 2 a protein expression by $\sim 80.67 \%$ was observed in si-Kif2a-transfected cells compared to si-NC and lipofectamine-only treated cells (Fig. $1 \mathrm{~A}$ and $\mathrm{B} ; \mathrm{P}=0.022$ ). These results indicated that si-Kif $2 \mathrm{a}$ had a gene-silencing effect targeting Kif2a at the protein level.

Apoptosis is induced by si-Kif2a in Tca8113 cells. Flow cytometric analysis revealed a significant increase in the percentage of apoptotic cells in Tca8113-Kif2a as compared to Tca8113-nonsense (NC) and Tca8113 cells (28.39 2.89 vs. $5.28 \pm 0.50$ and $3.73 \pm 0.57 \% ; \mathrm{P}<0.01)$. No significant difference was observed between Tca8113-NC and Tca8113. It was revealed that silencing Kif2a induced significant apoptosis in Tca8113 cells (Fig. 1C and D).

Gene expression patterns in si-Kif2a-transfected cells. Analysis of the gene expression profiles of the cells transfected with si-Kif2a and si-NC revealed that 744 genes were 
A

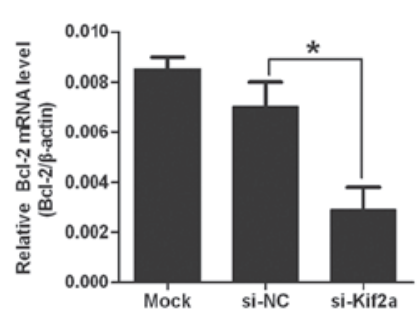

E
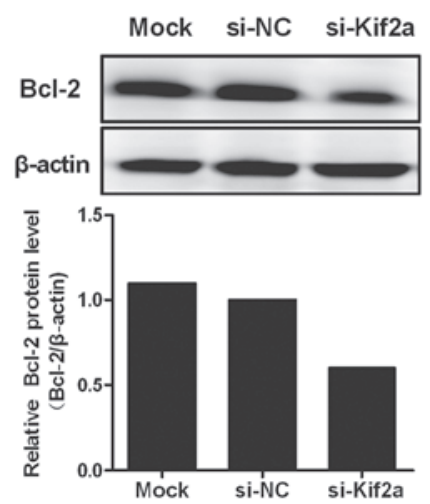

B

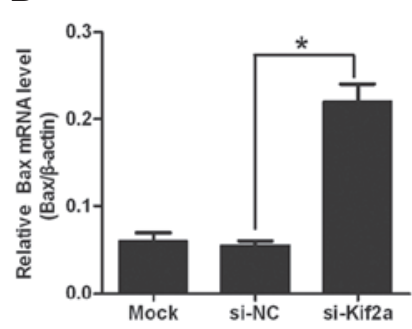

F
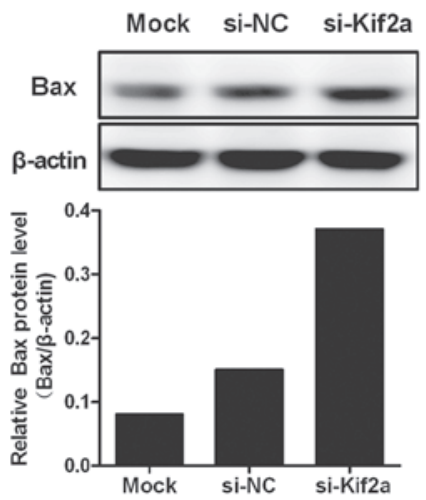

C

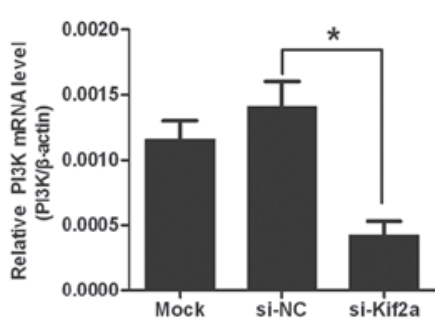

G
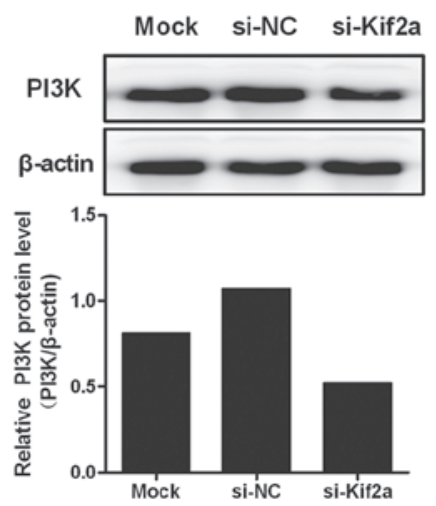

D

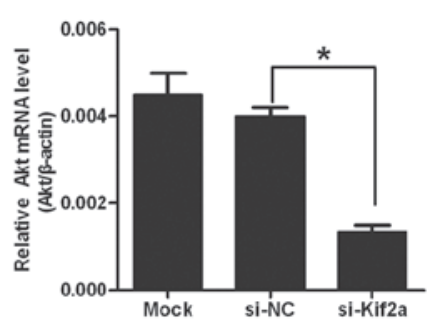

H

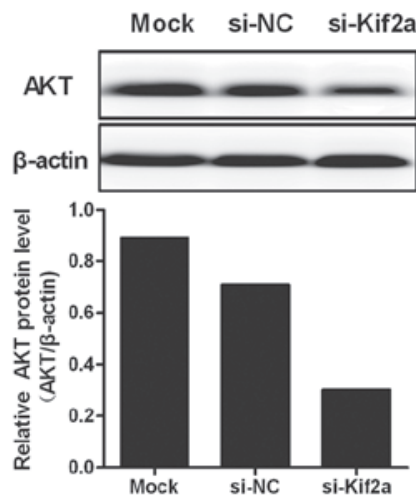

Figure 3. The expression of PI3K, Akt, Bcl-2 and Bax in different groups of Tca8113 cells at the mRNA level. The mRNA levels of (A) PI3K, (B) Akt, (C) Bcl-2 and (D) Bax were analyzed quantitatively by real-time polymerase chain reaction. The protein expression levels of (E) PI3K, (F) Akt, (G) Bcl-2 and $(\mathrm{H})$ Bax were analyzed quantitatively by western blot analysis. The level of PI3K, Akt and Bcl-2 mRNA in Tca8113-Kif2a decreased more clearly than Tca8113-nonsense or Tca8113 cells. However, the level of Bax in Tca8113-Kif2a cells was highest among the three groups ( $\mathrm{P}<0.05)$. The data are presented as the means \pm SD. Experiments were repeated $\geq 3$ times. Mock, lipofectamine only; si-NC, nonsense si-RNA; si-Kif2a, kif2a si-RNA.

A

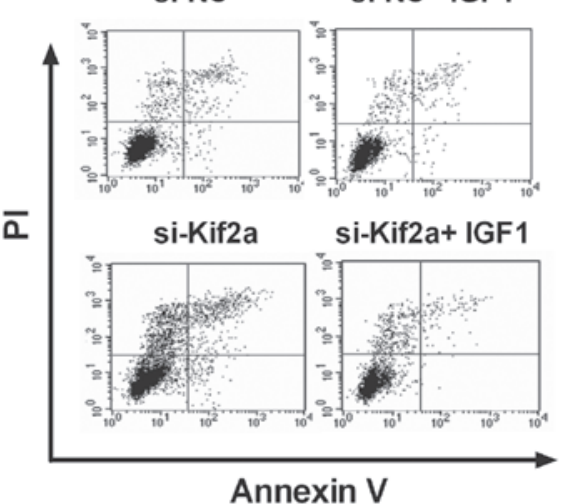

B

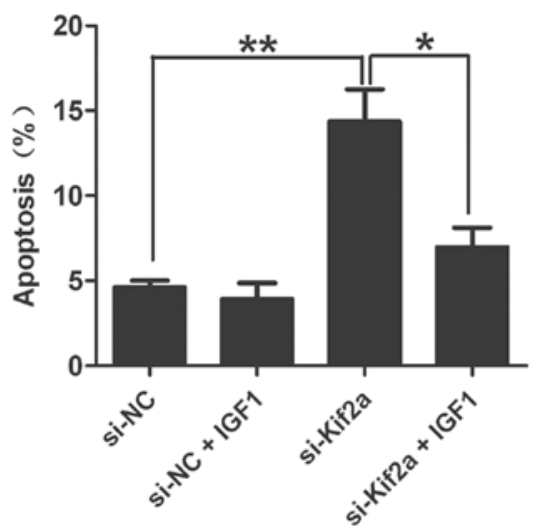

Figure 4. Insulin-like growth factor 1 (IGF-1) eliminated the upregulation of apoptosis of Tca8113-Kif2a cells. (A) Representative flow cytometry data. (B) The ratio of apoptosis of Tca8113-Kif2a cells was highest compared with other groups $\left({ }^{*} \mathrm{P}<0.05,{ }^{* *} \mathrm{P}<0.05\right)$. When the group Tca8113-Kif2a was added in IGF-1, the ratio of apoptosis was reduced. Data are presented as the means \pm SD. Experiments were repeated $\geq 3$ times. si-NC, nonsense si-RNA; si-Kif2a, kif $2 \mathrm{a}$ si-RNA.

upregulated and 1,282 genes were downregulated. The DAVID database indicated multiple biological pathways that appear to be enriched in the two cell groups. Most notably, the enrichment score for the protein processing in endoplasmic reticulum was significantly elevated. The enriched genes involved in the protein processing in endoplasmic reticulum included multiple members, such as BAX, SEC63, RAD23B, EIF2AK2, FBXO2, WFS1, BCAP31, MBTPS1, YOD1 and LMAN1. Of these genes, the expression of BAX gene has a significant change showing >4-fold upregulation in Tca8113-Kif2a cells (Fig. 2).
Expression of PI3K, Akt, Bcl-2 and Bax in different groups of Tca8113 cells. The mRNA level of PI3K, Akt, Bcl-2 and Bax were analyzed by real-time PCR. Data showed that PI3K, Akt and Bcl-2 in Tca8113-Kif2a cells decreased significantly compared to Tca8113-NC or Tca8113 cells (Fig. 3A-C). However, the level of Bax in Tca8113-Kif2a cells was the highest among the three groups (Fig. 3D). From the data, we hypothesize that silencing of Kif2a may be closely associated with the survival of Tca8113 cells by the PI3K/Akt signal pathway, particularly by regulating the ratio of $\mathrm{Bcl}-2 / \mathrm{Bax}$. 
A

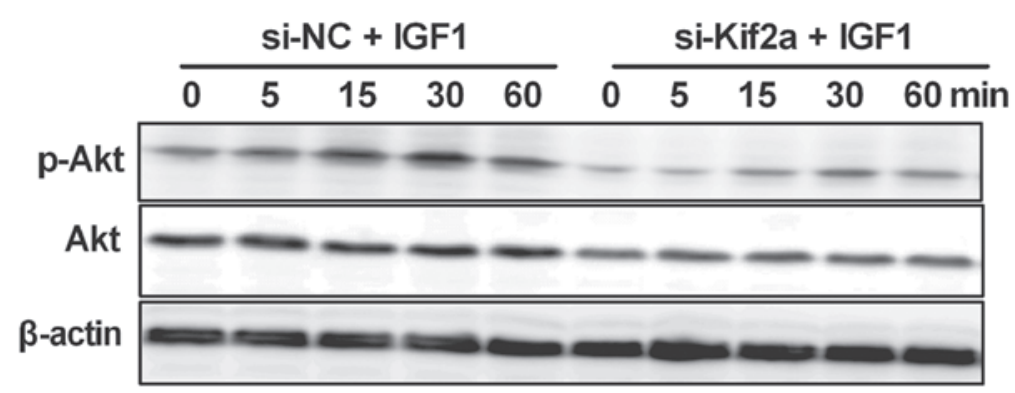

B

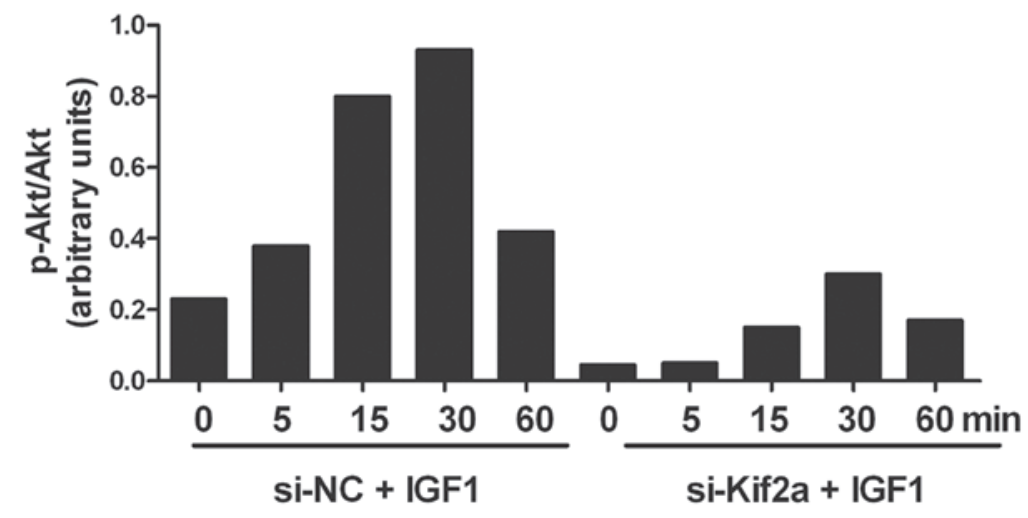

Figure 5. Apoptosis of Tca8113 cells is dependent on crosstalk between Kif2a and PI3K/Akt-signaling. (A) Western blot analysis of a time course of phospho-Akt and Akt in nonsense si-RNA (si-NC) and kif2a si-RNA (si-Kif2a) group with insulin-like growth factor 1 (IGF1), with actin as the loading control. (B) Histogram showing relative expression levels after normalization to $\beta$-actin. Data are mean \pm SD values from two independent experiments ( $=3$ ).

IGF-1 abrogates the upregulation of apoptosis of Tca8113-Kif2a cells. IGF-1 is the specific agonist of PI3K/Akt. When comparing the percentage of apoptotic cells in the si-Kif2a only group with the si-Kif2a and IGF-1 group, we found that IGF-1 effectively eliminated the upregulation of apoptosis in Tca8113-Kif2a cells (Fig. 4). It was revealed that silencing Kif2a significantly induced Tca8113 cell apoptosis through the PI3K/Akt pathway.

Silencing Kif2a induces apoptosis of Tca8113 cells by inhibiting the PI3K/Akt signaling pathway. In order to understand the molecular mechanisms of the apoptosis of Tca8113 cells by silencing Kif2a, we investigated PI3K/Akt signaling. The result showed strong phosphorylation of Akt at 30 min after adding IGF-1 in the si-NC group. In the experimental group, the Akt response was reduced by silencing Kif2a at all the indicated time points. However, the phosphorylation of Akt at 30 min after the addition of IGF-1 the si-Kif2a group also reached a peak (Fig. 5).

\section{Discussion}

In this study, to the best of our knowledge, the results demonstrate for the first time that silencing Kif2a leads to apoptosis of SCCOT cells and inhibits the PI3K/Akt signaling pathway, which may be one of the mechanisms responsible for inducing SCCOT cell apoptosis.

Several novel points arise from this study. First, we reported that silencing of Kif2a induces apoptosis of SCCOT cells. Previous studies on Kif2a were usually focused on its function and its mechanisms in depolymerizing microtubules. There has been no report on its role in regulating the apoptosis of tumor cells. Our data showed that silencing Kif2a increased the percentage of apoptotic cells.

Second, we provided evidence that Kif $2 \mathrm{a}$ is an upstream regulator of the PI3K/Akt pathway. Our finding that silencing Kif2a inhibits PI3K/Akt is significant since the overexpression and activation of PI3K/Akt is a frequent event in many types of human cancer $(5,11)$, although the mechanisms underlying its activation are not fully understood. Previously, it was reported that the EVI1 oncogene increases the sensitivity of colon cancer cells to taxol-mediated apoptosis through activation of PI3K/Akt (12) and ID-1 protects esophageal cancer cells from tumor necrosis factor- $\alpha$-induced apoptosis through activation of the PI3K/Akt signaling pathway (13). Whether a similar association exists between the PI3K/Akt pathway and other known oncogenes requires further investigation.

Third, the results showed that silencing Kif2a by si-RNA suppressed the PI3K/Akt signaling pathway and induced SCCOT cells to apoptosis, suggesting a potentially novel therapeutic strategy for SCCOT. Although PI3K/Akt is not the sole mediator of Kif2a-dependent cell survival, its functions in regulating cell cycle control, driving tumorigenesis and imparting chemoresistance to anticancer treatment make it an attractive target for cancer therapy (14). A number of candidate drugs targeting this pathway, such as inhibitors of PI3K, epidermal growth factor receptor, platelet-derived growth factor receptor and mammalian target of rapamycin (mTOR), as well as monoclonal HER2 antibody, have been studied. Rapamycin, an inhibitor of the Akt downstream mTOR, also has poor aqueous solubility and chemical stability, although it has significant anti-proliferative activity in several murine tumor systems (15). A rapamycin analog, CCI-779, with improved pharmaceutical properties and comparable efficacy, 
was approved in phase I and II of clinical studies; phase III trials are in progress (16). However, inhibitors of mTOR may not block all the functions of the PI3K/Akt pathway as they only affect one of the many downstream pathways of PI3K/Akt signaling. While new reagents targeting this pathway are being developed and tested, consideration should be given to targeting Kif2a as an alternative strategy in cancer therapy, since our previous study indicates that Kif2a has multiple effects on tumor progression including tumor growth, invasion and metastasis. From a therapeutic standpoint, since $\mathrm{Kif} 2 \mathrm{a}$ is overexpressed in SCCOT, but occurs at very low levels in normal tissues (3), inhibition of Kif2a should have very few side-effects on normal tissues. Furthermore, with the development of improved delivery systems in RNA interference technology and the recent success in the application of therapeutic si-RNA in non-human primates (17), RNAi-based therapeutic reagents targeting Kif $2 \mathrm{a}$ may be a promising alternative or adjunct to cytotoxic chemotherapy for SCCOT.

In conclusion, we confirmed that Kif $2 \mathrm{a}$ is an important molecule associated with the survival and progression of SCCOT cells. Thus, we hypothesize that Kif2a is a prognostic marker, or even a molecular target, for SCCOT therapy.

\section{Acknowledgements}

This study was supported by the National Natural Science Foundation of China (grant nos. 30772269, 81072202, 81271105 and 81202142) and Shandong Natural Science Foundation (no. Y2007C128).

\section{References}

1. Ganem NJ and Compton DA: The KinI kinesin Kif2a is required for bipolar spindle assembly through a functional relationship with MCAK. J Cell Biol 166: 473-478, 2004.

2. Ganem NJ, Upton K and Compton DA: Efficient mitosis in human cells lacking poleward microtubule flux. Curr Biol 15: 1827-1832, 2005.
3. Wang CQ, Qu X, Zhang XY, et al: Overexpression of Kif2a promotes the progression and metastasis of squamous cell carcinoma of the oral tongue. Oral Oncol 46: 65-69, 2010.

4. Bader AG, Kang S, Zhao L and Vogt PK: Oncogenic PI3K deregulates transcription and translation. Nat Rev Cancer 5: 921-929, 2005.

5. Brader S and Eccles SA: Phosphoinositide 3-kinase signalling pathways in tumor progression, invasion and angiogenesis. Tumori 90: 2-8, 2004

6. Yen CC, Chen YJ, Lu KH, et al: Genotypic analysis of esophageal squamous cell carcinoma by molecular cytogenetics and real-time quantitative polymerase chain reaction. Int J Oncol 23: 871-881, 2003.

7. Zhang G, Zhou X, Xue L, et al: Accumulation of cytoplasmic beta-catenin correlates with reduced expression of E-cadherin, but not with phosphorylated Akt in esophageal squamous cell carcinoma: immunohistochemical study. Pathol Int 55: 310-317, 2005.

8. Bian Y, Terse A, Du J, et al: Progressive tumor formation in mice with conditional deletion of TGF-beta signaling in head and neck epithelia is associated with activation of the PI3K/Akt pathway. Cancer Res 69: 5918-5926, 2009.

9. Iamaroon A and Krisanaprakornkit S: Overexpression and activation of Akt2 protein in oral squamous cell carcinoma. Oral Oncol 45: e175-e179, 2009.

10. Greenlee RT, Hill-Harmon MB, Murray T and Thun M: Cancer statistics, 2001. CA Cancer J Clin 51: 15-36, 2001.

11. Aggarwal BB: Nuclear factor-kappaB: the enemy within. Cancer Cell 6: 203-208, 2004.

12. Liu Y, Chen L, Ko TC, Fields AP and Thompson EA: Evi1 is a survival factor which conveys resistance to both TGFbetaand taxol-mediated cell death via PI3K/AKT. Oncogene 25: 3565-3575, 2006

13. Li B, Cheung PY, Wang X, Tsao SW, Ling MT, Wong YC and Cheung AL: Id-1 activation of PI3K/Akt/NFKB signaling pathway and its significance in promoting survival of esophageal cancer cells. Carcinogenesis 28: 2313-2320, 2007.

14. Xin $M$ and Deng X: Nicotine inactivation of the proapoptotic function of Bax through phosphorylation. J Biol Chem 280: 10781-10789, 2005.

15. Hennessy BT, Smith DL, Ram PT, Lu Y and Mills GB: Exploiting the PI3K/AKT pathway for cancer drug discovery. Nat Rev Drug Discov 4: 988-1004, 2005.

16. Morgensztern D and McLeod HL: PI3K/Akt/mTOR pathway as a target for cancer therapy. Anticancer Drugs 16: 797-803, 2005.

17. Zimmermann TS, Lee AC, Akinc A, et al: RNAi-mediated gene silencing in non-human primates. Nature 441: 111-114, 2006. 